\title{
Topological algebras with subadditive boundedness radius
}

\author{
M. SABET ${ }^{1}$ AND R. G. SANATI ${ }^{2}$ \\ ${ }^{1}$ Department of Mathematics \\ Payame Noor University, \\ Tehran, Iran. \\ ${ }^{2}$ Institute of Higher Education \\ of ACECR (Academic Center of Education and Culture Research), \\ Rasht branch, Iran. \\ sabet.majid@gmail.com, reza_sanaaty@yahoo.com
}

\begin{abstract}
Let $A$ be a topological algebra and $\beta$ a subadditive boundedness radius on $A$. In this paper we show that $\beta$ is, under certain conditions, automatically submultiplicative. Then we apply this fact to prove that the spectrum of any element of $A$ is non-empty. Finally, in the case when $A$ is a normed algebra, we compare the initial normed topology with the normed topology $\tau_{\beta}$, induced by $\beta$ on $A$, where $\beta^{-1}(0)=0$.
\end{abstract}

\section{RESUMEN}

Sea $A$ un álgebra topológica y $\beta$ un radio de acotamiento subaditivo en $A$. En este artículo mostramos que $\beta$ es, bajo ciertas condiciones, automáticamente submultiplicativo. Luego aplicamos este hecho para probar que el espectro de cualquier elemento de $A$ es no-vacío. Finalmente, en el caso cuando $A$ es una álgebra normada, comparamos la topología normada inicial con la topología normada $\tau_{\beta}$, inducida por $\beta$ en $A$, donde $\beta^{-1}(0)=0$.

Keywords and Phrases: Topological algebra, strongly sequential algebra, boundedness radius.

2020 AMS Mathematics Subject Classification: 46H05; 46H20 


\section{Introduction and Preliminaries}

A topological algebra is an algebra $A$ which is a topological vector space in such a way that the ring multiplication in $A$ is separately continuous. (i.e., continuous in each one of the two variables the latter operation being a map of $A \times A$ into $A$.)

If the multiplication of a given topological algebra $A$ is in both variables (i.e., jointly) continuous, we say that $A$ is a topological algebra with a continuous multiplication. (See e.g., [9, p. 4. Definition 1.1.])

In what follows the topological algebras cosidered are supposed with a continuous multiplication and to be Hausdorff.

Among topological algebras, the normed ones have been studied intensively by many mathematicians where the norm is used as a useful tool in measuring the distance between two elements. But, since 1940, there has been a considerable interest in investigating other topological algebras in absence of any norm and this led to the introduction of some famous topological algebras such as locally bounded algebras, locally convex algebras, locally multiplicatively convex algebras, et cetera.

The common idea to study a non-normed topological algebra, say $A$, is to substitute the role of a norm on $A$, which determines the topology, in an appropriate way. For instance, Aoki [2] proved that on a locally bounded algebra $A$, there is a p-norm generating the original topology on A. Later, Zelazko [12] applied this fact on locally bounded algebras, and obtained many classical results, known in the context of normed algebras. For example, in a complete locally bounded topological algebra $A$, the operator $x \mapsto x^{-1}$ on $\operatorname{Inv}(A)$ (the group of all invertible elements of $A$ ) is continuous. Also, the Cohen factorization theorem holds whenever $A$ has a bounded approximate identity. The role of norm in a locally convex topological algebra $A$ is played by a separating family of submultiplicative seminorms generating the topology on $A$. Recall that a seminorm is a non-negative real-valued function $p$ on $A$ such that

(i) $p(x+y) \leq p(x)+p(y)$

(ii) $p(\alpha x)=|\alpha| p(x)$

for all $x$ and $y$ in $A$ and $\alpha$ in $\mathbb{C}$.

We say that $p$ is submultiplicative seminorm if in addition $p(x y) \leq p(x) p(y)$ for all $x$ and $y$ in $A$.

Hence, there is a good motivation for mathematicians to extend notions and theorems from normed algebras to other topological algebras. A locally convex algebra is a topological algebra $A$ whose the underlying topological vector space is a locally convex space. The topology of a 
such topological algebra is determined by a family of (non-zero) seminorms. In the case when the seminorms are submultiplicative, $A$ is called a locally $m$-convex algebra.

Let $A$ be a unital topological algebra with the unit $e$ and $x \in A$. The spectrum of $x$, denoted by $\sigma(x)$, is defined

$$
\sigma(x)=\{\lambda \in \mathbb{C}: \lambda e-x \notin \operatorname{Inv}(A)\} .
$$

The spectral radius of $x$ is the defined as

$$
\rho(x)=\sup \{|\lambda|: \lambda \in \sigma(x)\}
$$

It is well known that $\rho(x)=\inf \left\{\left\|x^{n}\right\|^{\frac{1}{n}}: n \in \mathbb{N}\right\}$ for every element $x$ in a Banach algebra. Using this fact, Allan [1], introduced the notion of boundedness radius in a topological algebra $A$ as follows

$$
\beta(x)=\inf \left\{r>0:\left(\left(\frac{x^{n}}{r^{n}}\right)\right)_{n} \rightarrow 0\right\},(\inf \phi=+\infty) .
$$

Allan attempted to compare $\beta(x)$ with $\rho(x)$ and in one of the obtained results, shows that $\beta(x)$ is equal to $\rho(x)$ in a complete locally convex algebra [1, Theorem 3.12], specially, in a Banach algebra we have

$$
\rho(x)=\lim _{n \rightarrow \infty}\left\|x^{n}\right\|^{\frac{1}{n}}=\inf \left\{\left\|x^{n}\right\|^{\frac{1}{n}}: n \in \mathbb{N}\right\}=\beta(x) .
$$

Oubbi [11] investigated on $\rho$ and $\beta$ and compared them together. In a topological algebra $A$, he showed that $\rho \leq \beta$ if and only if $\sum x^{n}$ is convergent whenever $x \in A$ and $\beta(x)<1$. Also, he showed $\beta\left(x^{n}\right)=\beta(x)^{n}$ for all $n \in \mathbb{N}$ and $\beta(x y) \leq \beta(x) \beta(y)$ whenver $x, y \in A, x y=y x$. In any topological algebra, it is clear that $\beta(\lambda x)=|\lambda| \beta(x) \quad(x \in A, \lambda \in \mathbb{C})$.

Let $A \mathrm{~b}$ a topological algebra. The boundedness radius $\beta$ is said to be subadditive, if for each $x, y \in A, \beta(x+y) \leq \beta(x)+\beta(y)$. Moreover, $\beta$ is called submultiplicative whenever $\beta(x y) \leq$ $\beta(x) \beta(y)$.

Kinani, Oubbi and Oudadess [8], proved that in a unital and commutative locally convex algebra, $\beta$ is subadditive and submultiplicative. [8, Proposition II.9.]

In this paper, we show that in a topological algebra, if $\beta$ is finite and subadditive, then it is submultiplicative. (See Corollary 2.6.) Also, we refer to topological algebras in which $\beta$ defines a norm on them. (See Theorem 2.10 and Theorem 2.11.) Actually, we consider a topological algebra $A$ with boundedness radius $\beta$ such that $\beta$ satisfies the following conditions:

(1) $\beta^{-1}(0)=0$

(2) $\forall x \in A, \beta(x)<\infty$

(3) $\forall x, y, \beta(x+y) \leq \beta(x)+\beta(y)$. 


\section{The normed topology $\tau_{\beta}$ induced by the boundedness ra- $\operatorname{dius} \beta$}

In a locally convex algebra, amongst the important results is the following.

Theorem 2.1. Let $A$ be a unital and commutative locally convex algebra, then $\beta$ is subadditive.

Proof. See [8, Proposition II.9].

In this section, we first give an example to show that we can not drop commutativity in Theorem 2.1 and we also prove that locally convexity of an algebra is not sufficient for $\beta$ to be subadditive. Finally, we consider topological algebras in which $\beta$ is subadditive and we show that in such algebras, $\beta$ is automatically submultiplicative.

Example 2.2. Let $a=\left(\begin{array}{ll}0 & 0 \\ 1 & 0\end{array}\right)$ and $b=\left(\begin{array}{ll}0 & 1 \\ 0 & 0\end{array}\right)$ be elements of the noncommutative algebra $A=M_{2}(\mathbb{C})$. Since $a$ and $b$ are nilpotent elements of $A, \beta(a)=\beta(b)=0$, on the other hand $\beta(a+b)=1$ because $a+b=\left(\begin{array}{ll}0 & 1 \\ 1 & 0\end{array}\right)$ and so $(a+b)^{2}=\left(\begin{array}{ll}1 & 0 \\ 0 & 1\end{array}\right)=I$, now we have

$$
(\beta(a+b))^{2}=\beta\left((a+b)^{2}\right)=\beta(I)=1 .
$$

Which implies that $\beta(a+b)=1$. Hence $\beta(a+b) \not \leq \beta(a)+\beta(b)$.

This shows that we can not drop commutativity in Theorem 2.1 .

Let $A$ be a topological algebra and $B=A \times \mathbb{C}$ is equipped with the following multiplication

$$
\left(x_{1}, \alpha_{1}\right)\left(x_{2}, \alpha_{2}\right)=\left(\alpha_{1} x_{2}+\alpha_{2} x_{1}, \alpha_{1} \alpha_{2}\right) \quad\left(x_{1}, x_{2} \in A, \alpha_{1}, \alpha_{2} \in \mathbb{C}\right) .
$$

The unitization $B$ of $A$ is a topological algebra under the product topology of $B=A \times \mathbb{C}$ and we have:

Theorem 2.3. The boundedness radius $\beta$ is subadditive in $B$.

Proof. It is enough to show that for every $z=(x, \alpha) \in B, \beta(z)=|\alpha|$.

Let $z=(x, \alpha) \in B$. If $\alpha=0$ then $(x, 0)$ is a nilpotent element of $B$ and $\beta(z)=0=|\alpha|$. For $\alpha \neq 0$, suppose that $n \in \mathbb{N}, \varepsilon>0$. Let $r \in(|\alpha|,|\alpha|+\varepsilon)$ then $\frac{1}{r^{n}} \alpha^{n-1} \rightarrow 0$ and so $\frac{1}{r^{n}} \alpha^{n-1} x \rightarrow 0$. On the other hand, $(x, \alpha)^{n}=\left(n x \alpha^{n-1}, \alpha^{n}\right)$. Therefore

$$
\begin{aligned}
\frac{1}{(r+\varepsilon)^{n}}(x, \alpha)^{n} & =\frac{1}{(r+\varepsilon)^{n}}\left(n x \alpha^{n-1}, \alpha^{n}\right) \\
& =\left(n\left(\frac{r}{r+\varepsilon}\right)^{n} \frac{x \alpha^{n-1}}{r^{n}}, \frac{\alpha^{n}}{(r+\varepsilon)^{n}}\right) \rightarrow(0,0) .
\end{aligned}
$$


This shows that $\beta(z) \leq r+\varepsilon<|\alpha|+2 \varepsilon$, since $\varepsilon$ is arbitrary, $\beta(z) \leq|\alpha|$.

For the converse, note that there exists $r>0$ such that $r<\beta(x, \alpha)+\varepsilon$ and

$$
\frac{(x, \alpha)^{n}}{r^{n}}=\left(\frac{n x \alpha^{n-1}}{r^{n}}, \frac{\alpha^{n}}{r^{n}}\right) \rightarrow(0,0) .
$$

Hence, $\frac{\alpha^{n}}{r^{n}}$ convergent to zero. So $|\alpha|<r<\beta(x, \alpha)+\varepsilon$, it follows that $\beta(\alpha)=|\alpha|<\beta(z)+\varepsilon$. Thus $|\alpha| \leq \beta(z)$.

Definition 2.4. Let $A$ be an algebra. We say that a seminorm $p$ on $A$ has square property, if it is square-preserving, namely,

$$
p\left(x^{2}\right)=p(x)^{2} \text { for all } x \in A \text {. }
$$

Dedania in 1998 [6] proved the following theorem.

Theorem 2.5. Let $A$ be an algebra and a seminorm $p$ on $A$ which has the square property. Then $p$ is submultiplicative.

According to the terminology in $[4$, p. $437,(6)]$ and due to Theorem 2.5, a seminorm $p$. as in the latter theorem, is finally a uniform seminorm.

Corollary 2.6. Let $A$ be a topological algebra, such that $\beta(x)<\infty$ and $\beta(x+y) \leq \beta(x)+\beta(y)$ for all $x, y \in A$. Then $\beta$ is submultiplicative.

Proof. Since $\beta\left(x^{2}\right)=\beta(x)^{2}$, the assertion follows from Theorem 2.5.

Let $A$ be a topological algebra such that $\beta$ satisfies conditions (1)-(3) then $\beta$ is a norm on $A$ and by Corollary 2.6, $\beta$ is a submultiplicative norm. Through this section, $\tau_{\beta}$ denotes the topology, induced by $\beta$ on $A$. Now we face the following questions.

Question 1. Is there any topological algebra for which $\beta$ satisfies the conditions (1)-(3)?

Question 2. Let $(A, \tau)$ be a topological algebra for which $\beta$ satisfies the conditions (1)-(3). What is then the relation between $\tau$ and $\tau_{\beta}$ ?

Question 3. Let $A$ be a complete topological algebra such that $\beta$ satisfies the conditions (1)-(3). Does the normed algebra $\left(A, \tau_{\beta}\right)$ is a Banach algebra?

In what follows, we are going to answer to these questions.

Theorem 2.7. Let $A$ be a unital and commutative semisimple Banach algebra, then $\beta$ satisfies $(1)-(3)$.

Proof. Since $A$ is a commutative locally convex algebra, then, by [8, Lemma 2.9]

$$
\beta(x+y) \leq \beta(x)+\beta(y) \text { for all } x, y \text { in } A .
$$


Since $A$ is a Banach algebra then $A$ is locally bounded and using [3, Lemma 3.4], $\beta(x) \leq\|x\|$ for all $x$ in $A$, hence $\beta$ satisfies condition (2).

$A$ is a commutative semisimple Banach algebra, using Corollary 7.(iv) in [5] we have $\rho^{-1}(0)=$ 0 . Because $A$ is a Banach algebra, $\beta(x)=\rho(x)$ for each $x \in A$. So $\beta^{-1}(0)=0$. It follows that $\beta$ satisfies (1)-(3).

Theorem 2.7 gives a category of algebras satisfying conditions (1)-(3) and an affirmative answer to question 1.

Definition 2.8. A topological algebra $A$ is called strongly sequential if there is a neighborhood $U$ of zero such that, for all $x \in U,\left(x^{k}\right)_{k \in \mathbb{N}}$ converges to zero.

Lemma 2.9. Let $A$ be a topological algebra. Then $\beta$ is continuous at zero if and only if $A$ is strongly sequential.

Proof. See [3, Proposition 3.1].

In order to answer Question 2, we give the following theorem.

Theorem 2.10. Let $(A, \tau)$ be a topological algebra. Suppose that $\beta$ satisfies (1)-(3). Then $(A, \tau)$ is strongly sequential if and only if $\tau_{\beta} \subseteq \tau$.

Proof. Suppose $(A, \tau)$ is strongly sequential. By Lemma $2.9, \beta$ is continuous at zero. Since $\beta$ is subadditive, it is continuous on $A$. On the other hand, $\left\{\beta^{-1}\left(0, \frac{1}{n}\right): n \in \mathbb{N}\right\}$ is a local base for the normed topology $\tau_{\beta}$. Therefore $\tau_{\beta} \subseteq \tau$.

Conversely, let $x_{\alpha} \rightarrow 0$ in $\tau$. Since $\tau_{\beta} \subseteq \tau, x_{\alpha}$ converges to zero in $\tau_{\beta}$ which implies that $\beta\left(x_{\alpha}\right) \rightarrow 0$. Hence $\beta$ is continuous at zero and, again by Lemma $2.9, A$ is strongly sequential.

In order to answer Question 3, first we characterize a topological algebra for which $\left(A, \tau_{\beta}\right)$ is a Banach algebra and then we apply this characterization to give a negative answer to Question 3.

It is well known (See e.g., [10, p. 41]) that in a commutative $C^{*}$-algebra, the unique $C^{*}$-norm is the spectral norm i.e.

$$
\|x\|=\rho(x) \quad(x \in A)
$$

On the other hand, for each $x \in A, \rho(x)=\beta(x)$ and so $\beta$ is indeed the $C^{*}$-norm on $A$. Thus $\left(A, \tau_{\beta}\right)$ is a complete normed algebra whenever $A$ is a $C^{*}$-algebra. The following theorem gives a more general characterization of topological algebras for which $\left(A, \tau_{\beta}\right)$ is complete.

In the sequel, by an $F$-algebra we mean a completely metrizable topological algebra. 
Theorem 2.11. Let $(A, \tau)$ be a strongly sequential F-algebra. Suppose that $\beta$ satisfies (1)-(3). Then $\left(A, \tau_{\beta}\right)$ is a Banach algebra if and only if $\tau_{\beta}=\tau$.

Proof. If $\tau_{\beta}=\tau$, then $\left(A, \tau_{\beta}\right)$ is a Banach algebra, trivially. For the converse, suppose that $\left(A, \tau_{\beta}\right)$ is a Banach algebra. By the assumption, $(A, \tau)$ is a strongly sequential $F$-algebra, so, Theorem 2.10 implies, $\tau_{\beta} \subseteq \tau$. From the open mapping theorem, one immediately gets that $\tau_{\beta}=\tau$.

The following example shows that the answer of Question 3 is not affirmative.

Example 2.12. Let $A$ be the set of $C^{1}$-functions on the interval $[0,1]$ and $f \in A$. Then $A$ is a semisimple commutative Banach algebra where the norm on $A$ is given by $\|f\|=\|f\|_{\infty}+\left\|f^{\prime}\right\|_{\infty}$ (See [10, p. 10, Example 1.2.6]).

Thus $\beta$ satisfies (1)-(3) and so $\left(A, \tau_{\beta}\right)$ is normed algebra. If $\left(A, \tau_{\beta}\right)$ is complete, then $A$ is a Banach algebra, also it is a strongly sequential algebra (See e.g., [7, p. 58, Example 3.26]). Now, by Theorem 2.11, $\tau_{\beta}=\tau$. Let $x$ be the identity map on $[0,1]$. Since $\beta(x)=1$, the sequence $\left(\frac{x^{n}}{n}\right)_{n}$ convergence to zero in $\tau_{\beta}$-topology. But $\frac{x^{n}}{n} \nrightarrow 0$ in the original topology $\tau$. This is a contradiction and so $\left(A, \tau_{\beta}\right)$ is not complete.

As we mentioned, if $\beta$ satisfies (1)-(3), then it is an algebraic norm on $A$. But in the absence of one of the properties (1)-(3), $\beta$ is not a norm necessarily. In what follows, we concentrate to study topological algebras for which $\beta$ is not a norm.

Lemma 2.13. Let $(A,\|\|)$ be a normed algebra such that, $\|a\|^{2}=\left\|a^{2}\right\|$ for all $a \in A$. Then $A$ is commutative.

Proof. See [5, p. 77, Corollary 8, see also the comments after it].

Theorem 2.14. Let $A$ be a topological algebra such that

(1) $\beta(x)<\infty(x \in A)$

(2) $\beta(x+y) \leq \beta(x)+\beta(y)(x, y \in A)$.

Then $\beta(x y-y x)=0$ for all $x, y$ in $A$.

Proof. Let $N=\{x \in A, \beta(x)=0\}$. It is clear that $N$ is an ideal in $A$ and $A / N$ is a normed algebra with the norm $\|x+N\|=\beta(x)$. Since $\beta$ has the square property, $\left\|(x+N)^{2}\right\|=\|x+N\|^{2}$ for all $x$ in $A$. Now, according to Lemma 2.13, the normed algebra $A / N$ is commutative. Hence, $(x+N)(y+N)=(y+N)(x+N)$ for all $x, y$ in $A$ which means that $\beta(x y-y x)=0$.

Corollary 2.15. Let $A$ be a topological algebra and $\beta$ satisfies (1)-(3), then $A$ is commutative. 
Proof. Since $\beta\left(x^{2}\right)=\beta(x)^{2}$, the assertion follows from Lemma 2.13 or Theorem 2.14.

Lemma 2.16. Let $A$ be a Banach algebra and $x, y \in A$. If $x y=y x, x o x=y o y(x o y=x+y-x y)$ and $\beta(x+y)<2$, then $x=y$.

Proof. See [5, p. 44, Lemma 12].

Lemma 2.17. Let $A$ be a normed algebra and $x, y \in A$. If $x y=y x, x o x=y o y$ and $\beta(x+y)<2$, then $x=y$.

Proof. Let $\tilde{A}$ be the completion of $A$. Suppose that $T$ is an isometric isomorphism of $A$ onto $\tilde{A}$. Since $(T x)(T y)=(T y)(T x),(T x) o(T y)=(T y) o(T x)$ and $\beta_{\tilde{A}}(T x+T y)=\beta_{\tilde{A}}(T(x+y))=\beta_{A}(x+y)<2$ by Lemma $2.16, T x=T y$ and so $x=y$.

Theorem 2.18. Let $A$ be a topological algebra and $x, y \in A$ such that $x o x=y o y$ and $\beta(x+y)<2$. If $\beta$ is finite and subadditive, then $\beta(x-y)=0$.

Proof. Assume that $N=\{x \in A, \beta(x)=0\}$. By the proof of Theorem 2.14, $A / N$ is a normed algebra under the norm $\|x+N\|=\beta(x)$. Then

$$
(x+N) o(x+N)=(y+N) o(y+N),
$$

and,

$$
\begin{aligned}
\beta_{A / N}((x+N)+(y+N)) & =\beta_{A / N}((x+y)+N) \\
& \leq\|(x+y)+N\|=\beta(x+y)<2
\end{aligned}
$$

Applying Lemma 2.17, $x+N=y+N$ which means that $\beta(x-y)=0$.

Theorem 2.19. Let $A$ be a topological algebra such that

(i) $\beta(x+y) \leq \beta(x)+\beta(y)(x, y \in A)$

(ii) $\beta(x)<\infty(x \in A)$.

Then $\sigma_{A}(x) \neq \emptyset$ for all $x \in A$.

Proof. Let $N=\{x \in A, \beta(x)=0\}$. Since $A / N$ is a normed algebra, the spectrum of any of its elements is non-empty. (See e.g., [5, p. 22. Theorem 7.]) On the other hand, the canonical map $\pi: A \rightarrow A / N$ is an algebraic homomorphism and so $\emptyset \neq \sigma_{A / N}(\pi(x)) \subseteq \sigma_{A}(x)$ for each $x \in A$. (See e.g., [5, p. 48. Proposition 9]) This completes the proof. 


\section{References}

[1] G. R. Allan, A spectral theory for locally convex algebras, Proc. London Math. Soc. (3) vol. 15, pp. 399-421, 1965.

[2] T. Aoki, Locally bounded linear topological spaces, Proc. Imp. Acad. Tokyo, vol. 18, pp. 588$594,1942$.

[3] E. Ansari-Piri, M. Sabet and S. Sharifi, A class of complete metrizable $Q$-algebras, Sci. Stud. Res. Ser. Math. Inform. vol. 26, no. 1, pp. 17-24, 2016.

[4] S. J. Bhatt, A seminorm with square property on a Banach algebra is submultiplicative, Proc. Amer. Math. Soc. vol. 117, no. 2, pp. 435-438,1993.

[5] F. F. Bonsall and J. Duncan, Complete normed algebras, Springer-Verlag, New York, 1973.

[6] H. V. Dedania, A seminorm with square property is automatically submultiplicative, Proc. Indian Acad. Sci. Math. Sci. vol. 108 1998, no. 1, pp. 51-53, 1998.

[7] T. Husain, Multiplicative functionals on topological algebras, Research Notes in Mathematics, 85, Pitman (Advanced Publishing Program), Boston, MA, 1983.

[8] A. El Kinani, L. Oubbi and M. Oudadess, Spectral and boundedness radii in locally convex algebras, Georgian Math. J. vol. 5, no. 3, pp. 233-241, 1998.

[9] A. Mallios, Topological algebras. Selected topics, North-Holland Mathematics Studies, 124, North-Holland Publishing Co., Amsterdam, 1986.

[10] G. J. Murphy, $C^{*}$-algebras and operator theory, North-Holland. 1990.

[11] L. Oubbi, Further radii in topological algebras, Bull. Belg. Math. Soc. Simon Stevin vol. 9, no. 2, pp. 279-292, 2002.

[12] W. Żelazko, On the locally bounded and $m$-convex topological algebras, Studia Math. vol. 19, pp. 333-356, 1960. 